\title{
Bimodal Character of Stress Transmission in Granular Packings
}

\author{
Farhang Radjai, ${ }^{1}$ Dietrich E. Wolf, ${ }^{1}$ Michel Jean, ${ }^{2}$ and Jean-Jacques Moreau ${ }^{3}$ \\ ${ }^{1}$ Theoretische Physik, FB 10, Gerhard Mercator-Universität, 47048 Duisburg, Germany \\ ${ }^{2}$ IMT-LMA, Technopôle de Château Gombert, 13451 Marseille Cedex 20, France \\ ${ }^{3}$ LMGC, Université de Montpellier II, Place Eugène Bataillon, 34095 Montpellier Cedex 05, France
}

The correlation between contact forces and the texture of a packing of rigid particles subject to biaxial compression is analyzed by means of numerical simulations. Four different aspects are investigated: stress tensor, dissipation due to friction, angular distribution of forces, and fabric tensor characterizing the anisotropy of the texture. All of them provide evidence that the contact network can be decomposed unambiguously into two subnetworks with complementary mechanical properties.

The plasticity of a packing of rigid spheres is maybe the simplest example in which the dynamics is dominated by topological constraints: Forces are transmitted only through the interparticle contacts. This leads to strong inhomogeneities of the forces [1-4]. Moreover, an initially isotropic packing develops an anisotropic contact network under shear, because new contacts are formed along the major principal axis of the strain-rate tensor, while some are lost perpendicular to it [5-7]. This geometrical anisotropy leads in turn to a mechanical anisotropy of the contact forces. Both the geometrical and the mechanical anisotropy enter the expression of the stress tensor and are thus essential for the resistance of a granular medium to shear $[8,9]$.

In this Letter, we analyze the transmission of stress in a two-dimensional dense packing of rigid spheres by taking for the first time both the inhomogeneity of the forces and the anisotropy of the texture into account. It will be shown that the forces belong to two distinct classes which contribute differently to anisotropy, stress, and dissipation. This bimodal character of the force network is quite naturally suggested by the observation of the "buckling" of strong force chains supported by weak lateral forces during shear [10]. The results presented in this Letter provide an unambiguous demonstration of this intuitive picture for all the aspects considered.

The main idea of our analysis is to evaluate internal variables such as the geometrical anisotropy for subsets of contacts with a given absolute value of the force. Thereby important aspects of the inhomogeneity of the system can be taken into account. For example, the contribution of contact chains with strong forces may be evaluated separately from the rest of the packing. This is, however, not practical because of the bad statistics of contacts within a small force interval. Instead, we consider the subset of contacts which carry a force lower than a given cutoff $\xi$. We shall refer to this subset as the " $\xi$-network." The variation of a quantity evaluated for the $\xi$-network as $\xi$ is varied from 0 to the maximal force in the system allows us then to estimate its correlation with the contact force.
For the numerical simulations, we used the relatively new method of contact dynamics. This method allows us to integrate the equations of motion for multicontact systems composed of rigid bodies with Coulombian friction. The method tackles the nonsmooth character of the interactions with no resort to regularization schemes often used in numerical algorithms for granular systems. An account of the mathematical basis and the discretization procedure of this approach can be found in [11].

The simulation was carried out for a two-dimensional system with 4012 circular particles contained in a frame of four rigid walls. The radii were uniformly distributed between 3.8 and $7.5 \mathrm{~mm}$. The particle-particle and particlewall coefficients of friction were 0.5 and zero, respectively. No gravity acted on the particles. The sample was biaxially compressed (see Fig. 1) by imposing a constant velocity of $1 \mathrm{~cm} / \mathrm{s}$ on the upper wall. The left wall was free to move under a horizontal confining force of $500 \mathrm{~N}$. The initial sample was prepared with an isotropic contact network. As a consequence of compression the amplitude of anisotropy and the stress ratio $Q=\left(\sigma_{1}-\sigma_{2}\right) /\left(\sigma_{1}+\right.$ $\sigma_{2}$ ) increased, where $\sigma_{1}$ and $\sigma_{2}$ are the principal values of the stress tensor. We investigate below several quantities as a function of $\xi$ at $Q=0.18$.

The texture is characterized by the probability density $E(\theta, \xi)$ of finding a contact with direction $\theta$ in the $\xi$ network. In general the first deviatoric component in the Fourier expansion of $E$ provides an adequate measure of geometrical anisotropy [12]:

$$
E(\theta, \xi) \approx \frac{1}{2 \pi}\left\{1+A_{c}(\xi) \cos 2\left[\theta-\theta_{c}(\xi)\right]\right\} .
$$

The parameter $A_{c}$ defines the amplitude of anisotropy, and $\theta_{c}$ is its principal direction. For the calculation of these parameters from the numerical data, it is convenient to use the "fabric tensor" defined by $\phi_{i j}=\left\langle n_{i} n_{j}\right\rangle$, where $n_{i}$ is the $i$ component of the unit vector along the contact direction and the average is taken over all contacts in the $\xi$-network. Using (1) to evaluate the average of $n_{i} n_{j}$, it is easy to see that $\phi_{1}-\phi_{2}=A_{c} / 2$, where $\phi_{1}$ and $\phi_{2}=1-\phi_{1}$ are 


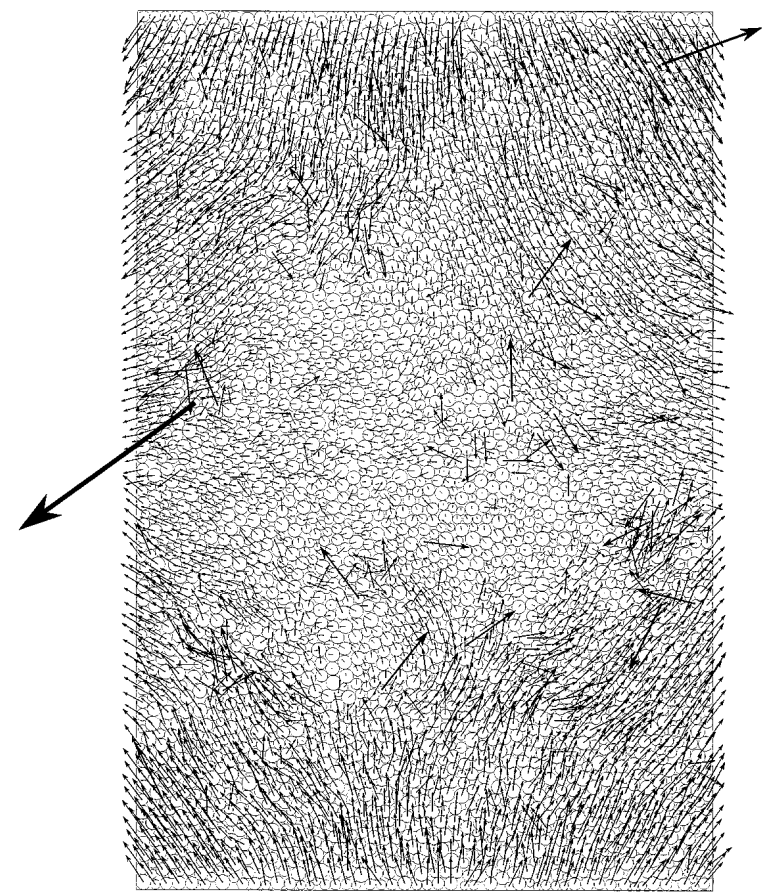

FIG. 1. Velocity field in the center-of-mass frame during the biaxial compression.

the eigenvalues of the fabric tensor. $\theta_{c}$ and $\theta_{c}+\pi / 2$ are the directions of the corresponding eigenvectors.

We found that the principal directions of the fabric tensor in the $\xi$-network coincide with those of the strainrate tensor irrespective of the value of $\xi$. Therefore we set $\theta_{c}(\xi)=\theta_{c}(\infty)=0$, i.e., the direction of the axis of compression in (1). Then a positive $A_{c}$ indicates that the direction of anisotropy is parallel to the axis of compression, whereas a negative $A_{c}$ corresponds to the orthogonal direction. Figure 2 shows the amplitude of geometrical anisotropy $A_{c}$ in the $\xi$-network as a function of $\xi$. For large $\xi$ it approaches the geometrical anisotropy

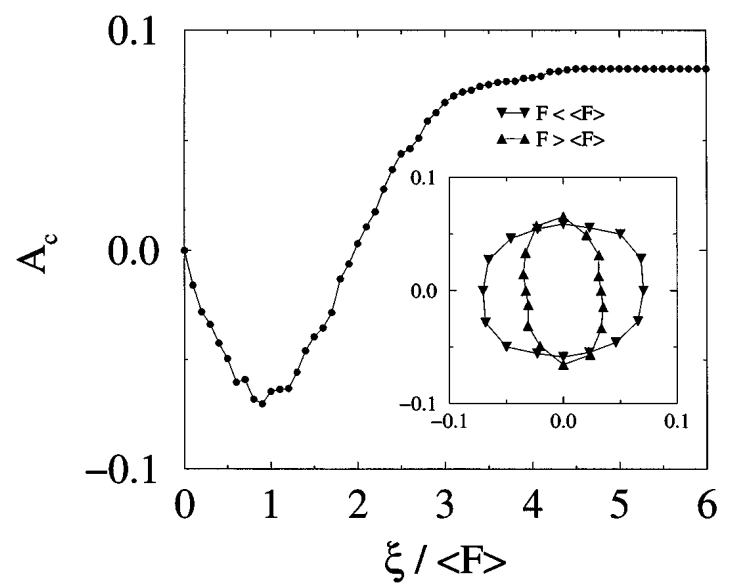

FIG. 2. Amplitude of geometrical anisotropy $A_{c}$ in the $\xi$ network as a function of $\xi$ normalized with respect to the average force $\langle F\rangle$; see text. The inset shows the polar diagrams of the probability density $E$ of contact directions for "weak" contacts $(F<\langle F\rangle)$ and for "strong" contacts $(F>\langle F\rangle)$. of the whole system. The anisotropy of the network complementary to the $\xi$-network is given by $A_{c}(\infty)-$ $A_{c}(\xi)$ and can be obtained from Fig. 2.

Surprisingly, the direction of anisotropy is orthogonal to the axis of compression $\left(A_{c}<0\right)$ for weak forces (small $\xi)$. The anisotropy becomes more pronounced as $\xi$ increases, and reaches a maximum for $\xi=\langle F\rangle$, where $\langle F\rangle$ is the average force in the system. When $\xi$ is increased beyond $\langle F\rangle, A_{c}$ becomes less negative and finally changes sign. This shows that contacts which carry a force larger than the average force ("strong contacts") are preferentially oriented parallel to the axis of compression. Although these are less than $40 \%$ of all contacts, their positive contribution to $A_{c}$ overcompensates the negative contribution of the contacts with a force lower than the average force ("weak contacts"). This means that the strong network (composed of strong contacts) is more anisotropic than the weak network (composed of weak contacts), as shown in the inset of Fig. 2.

The orthogonal anisotropy of the weak network cannot be simply understood as a result of the process of loss and gain of contacts induced by the deformation. The latter predicts only a positive anisotropy, i.e., parallel to the axis of compression. Our result, Fig. 2, proves that a sheared granular packing is not only inhomogeneous with respect to the forces, but also with respect to the geometrical anisotropy, and that these inhomogeneities are correlated.

A similar analysis can now be applied to investigate the mechanical anisotropy of the average normal force $F_{n}(\theta, \xi)$ and the average friction force $F_{t}(\theta, \xi)$ as a function of the contact direction. As for $E$, a second order Fourier expansion provides an adequate representation:

$$
\begin{aligned}
F_{n}(\theta, \xi) & =(\langle F\rangle / 2 \pi)\left\{1+A_{n}(\xi) \cos 2\left[\theta-\theta_{f}(\xi)\right]\right\}, \\
F_{t}(\theta, \xi) & =(\langle F\rangle / 2 \pi) A_{t}(\xi) \sin 2\left[\theta-\theta_{f}(\xi)\right],
\end{aligned}
$$

where $A_{n}$ and $A_{t}$ are the magnitudes of mechanical anisotropy. The analytical form of $F_{t}$ results from the fact that the spherical component of $F_{t}$ is zero due to static equilibrium and its principle axes are rotated to those of $F_{n}$ by an angle of $\pi / 4$. Again we found that the principal directions are independent of $\xi$, so that we shall set $\theta_{f}=0$ in the following.

For the calculation of $A_{n}$ and $A_{t}$ we introduce two tensors $\chi_{i j}^{(n)}=(1 /\langle F\rangle)\left\langle F_{n} n_{i} n_{j}\right\rangle$ and $\chi_{i j}^{(t)}=(1 /\langle F\rangle)\left\langle F_{t} t_{i} n_{j}\right\rangle$, where $t_{i}$ is the $i$ component of the unit vector $t$ orthogonal to $\boldsymbol{n}$ and such that $(\boldsymbol{n}, \boldsymbol{t})$ preserves the same parity for all contacts. It can then be shown that $\left(\chi_{1}^{(n)}-\chi_{2}^{(n)}\right) /$ $\left(\chi_{1}^{(n)}+\chi_{2}^{(n)}\right)=(1 / 2)\left(A_{c}+A_{n}\right)$ and $\left(\chi_{1}-\chi_{2}\right) /\left(\chi_{1}+\right.$ $\left.\chi_{2}\right)=(1 / 2)\left(A_{c}+A_{n}+A_{t}\right)$, where $\chi_{1}^{(n)}$ and $\chi_{2}^{(n)}$ are the principal values of $\boldsymbol{\chi}^{(n)}$ and $\chi_{1}$ and $\chi_{2}$ are those of $\boldsymbol{\chi}=$ $\boldsymbol{\chi}^{(n)}+\boldsymbol{\chi}^{(t)}$. In Fig. $3, A_{n}$ and $A_{t}$ in the $\xi$-network are plotted as a function of $\xi$. The two parameters remain positive, i.e., the mechanical anisotropy is always oriented along the axis of compression. However, the contribution of normal forces to the total anisotropy begins to increase 


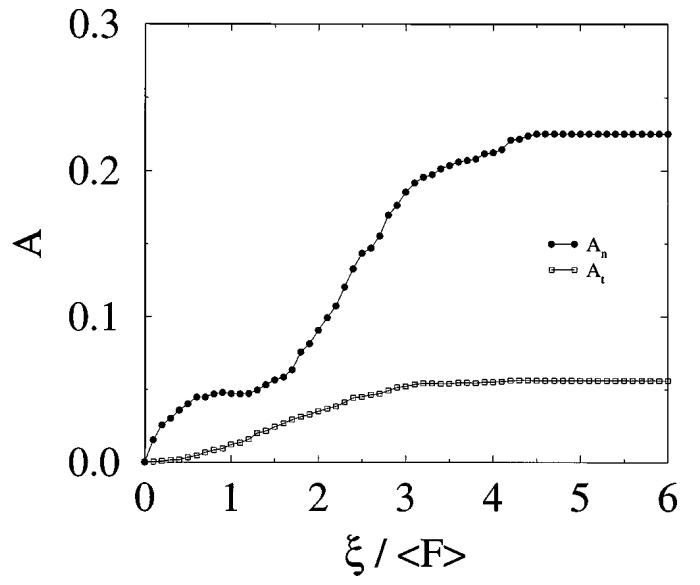

FIG. 3. Amplitudes of mechanical anisotropy $A_{n}$ and $A_{t}$ in the $\xi$-network as a function of $\xi /\langle F\rangle$.

significantly only after $\xi=\langle F\rangle$, where both $A_{n}$ and $A_{t}$ have an inflection point.

The physical importance of mechanical anisotropy becomes clear when it is considered in connection with the stress tensor. The stress tensor $\boldsymbol{\sigma}$ for a granular system in a quasistatic state is given by [13]

$$
\sigma_{i j}=\rho\left\langle F_{i} d_{j}\right\rangle,
$$

where $\rho$ is the number of contacts per unit volume, $F_{i}$ is the $i$ component of the contact force $\boldsymbol{F}=F_{n} \boldsymbol{n}+F_{t} \boldsymbol{t}$, and $d_{j}$ is the $j$ component of the intercenter vector joining the centers of two particles in contact. For spherical particles, we have $\boldsymbol{d}=\boldsymbol{d} \boldsymbol{n}$. Neglecting the weak correlation between $d$ and $F$, we get $\sigma_{i j}=\rho\langle d\rangle\left\langle F_{i} n_{j}\right\rangle$. Now, introducing the two components of $\boldsymbol{F}$ into this expression and comparing with the expression of $\boldsymbol{\chi}$, we see that $\boldsymbol{\sigma}=\rho\langle d\rangle\langle F\rangle \boldsymbol{\chi}$, and accordingly [8]

$$
Q(\xi)=\frac{1}{2}\left[A_{c}(\xi)+A_{n}(\xi)+A_{t}(\xi)\right] .
$$

In Fig. 4, both the stress ratio $Q$ and the sum $(1 / 2)\left(A_{c}+\right.$ $\left.A_{n}+A_{t}\right)$ are displayed as a function of $\xi$. We see that Eq. (4) holds for all $\xi$ with very weak deviations due to a weak correlation between $d$ and $F$.

Figure 4 reveals an unexpected property of the stress tensor: the shear stress $Q$ for all forces lower than the average force is negligibly small compared to the total deviatoric load $Q(\infty)$ sustained by the system. Those forces contribute only $28 \%$ of the average pressure $\left(\sigma_{1}+\right.$ $\left.\sigma_{2}\right) / 2$ in the medium. This means that the weak network behaves essentially like an interstitial liquid, whereas the strong forces carry the whole deviatoric load and in this respect behave like a solid. Furthermore, Eq. (4) shows that this property is related to a compensation between the negative anisotropy of fabric and the positive anisotropy of forces, so that $A_{c}+A_{n}+A_{t} \simeq 0$ for forces lower than the average.

Another aspect of stress transmission in a granular packing is the appearance of chainlike structures of relatively strong forces. This observation is suggestive of long-range

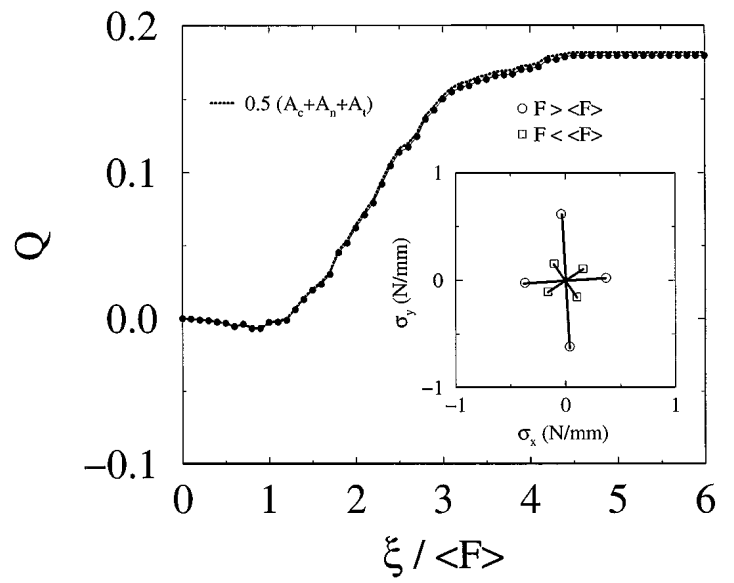

FIG. 4. Stress ratio $Q$ and the sum $0.5\left(A_{c}+A_{n}+A_{t}\right)$ in the $\xi$-network as a function of $\xi /\langle F\rangle$. The inset shows the eigenvalues and the directions of the stress tensor for weak $(F<\langle F\rangle)$ and strong $(F>\langle F\rangle)$ contacts. The orientation of the weak tensor is irrelevant since its deviatoric component is nearly zero.

correlations over a scale far larger than the particle size. In our numerical experiments, we can check these correlations as a function of $\xi$ in the complementary network to the $\xi$-network. At large values of $\xi$, say $\xi>2\langle F\rangle$, strong contacts are distributed in the form of relatively isolated clusters. As $\xi$ is decreased, these clusters grow [see Fig. 5(a)] and finally at $\xi=\langle F\rangle$ there is directed percolation along the axis of compression [Fig. 5(b)]. The fact that the whole deviatoric load is supported by a percolating network of strong chains makes it plausible that buckling of the directed chains under the action of compression occurs. Their stability then requires lateral forces in the complementary network. This is the origin of a negative geometrical anisotropy in the weak network. This mechanism goes with a peculiar scheme of the "mobilization" of friction, defined by the ratio $\eta=\left|F_{t}\right| / F_{n}$. Figure 6 shows the proportion of sliding contacts to the total number of contacts in the $\xi$-network as a function of $\xi$. At sliding contacts the friction force takes its maximum mobilized value, $\eta=\mu$, where $\mu$ is the coefficient of friction. At nonsliding contacts, particles roll over one another and $\eta<\mu$ [14]. Almost $8 \%$ of contacts are sliding in the whole volume of the system at $Q=0.18$, and Fig. 6 shows that $96 \%$ of them are in the weak network for $\xi=\langle F\rangle$. In other words, almost the whole dissipation by friction occurs at contacts bearing a force lower than the average force. Almost all contacts with a force larger than the average, corresponding to the buckling chains, are thus nonsliding.

In all the cases briefly discussed above, the average force appears as a characteristic force separating two complementary networks: a "load-bearing" percolating network of contacts carrying a force larger than the average force, and a "dissipative" network of contacts carrying a force smaller than the average force. The load-bearing network carries the whole deviatoric load, while the dissipative subnetwork 


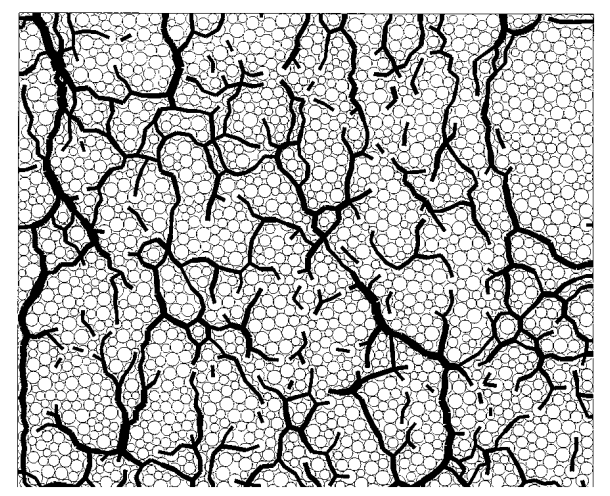

(a)

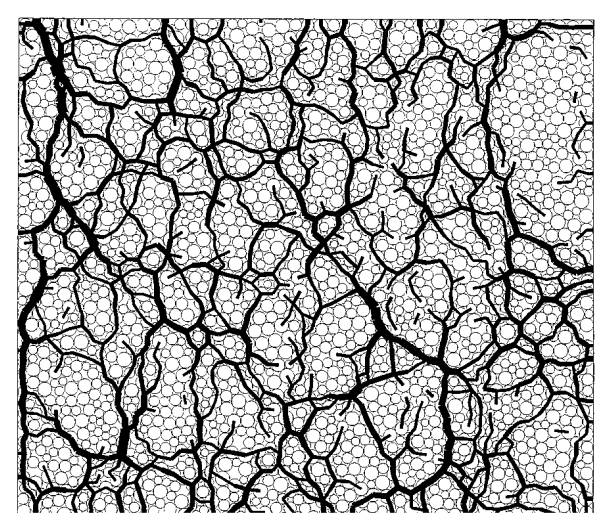

(b)

FIG. 5. The forces $F$ for (a) $F>1.3\langle F\rangle$ and (b) $F>\langle F\rangle$, where $\langle F\rangle$ is the average force, in the upper halves of the sample. The line thickness is proportional to the force.

contributes only to the average pressure. All contacts within the load-bearing network are nonsliding, whereas nearly the whole dissipation due to sliding takes place inside the dissipative network. The load-bearing subnetwork carries a direct geometrical anisotropy induced by shear, but it gives rise via buckling to an indirect anisotropy inside the dissipative network with a preferred direction orthogonal to the major principal direction of the stress tensor.

For all the variables studied here, this distinction between the two networks disappears in the particular case where the shear stress is zero. But it still holds for the statistical distribution $P_{F}$ of forces. $P_{F}$ is a power law with a weak negative exponent for forces lower than the average force, and an exponentially decreasing function for forces larger than the average [3]. The same behavior is observed in 3D systems as well [15].

The central message is that the inhomogeneous distribution of forces on the particle scale does not average out at the macroscopic level. It induces a bimodal behavior for the macroscopic variables of interest. More particularly, we find that a more precise description of stress transmission in a dry granular packing requires two stress tensors

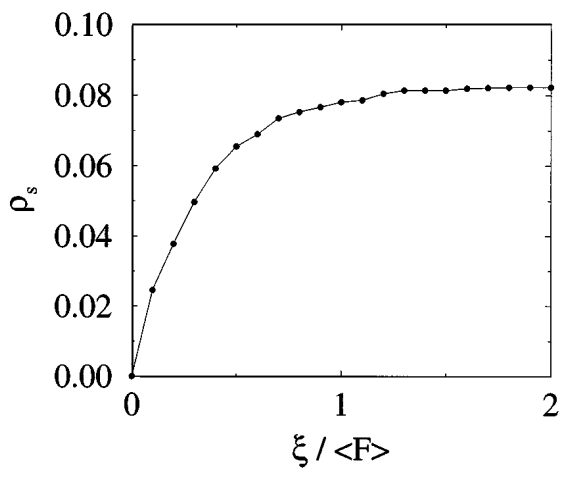

FIG. 6. Proportion $\rho_{s}$ of sliding contacts to the total number of contacts in the $\xi$-network as a function of $\xi /\langle F\rangle$.

corresponding to two complementary phases. This property of a quasistatic granular medium is in contrast to both liquids and solids.

It is a great pleasure to acknowledge many stimulating discussions with S. Roux and L. Brendel.

[1] P. Dantu, in Proceedings of the 4th International Conference on Soil Mechanics and Foundations Engineering (Butterworths Scientific Publications, London, 1957).

[2] C.-h. Liu, S. R. Nagel, D. A. Schecter, S. N. Coppersmith, S. Majumdar, O. Narayan, and T. A. Witten, Science 269, 513 (1995).

[3] F. Radjai, M. Jean, J. J. Moreau, and S. Roux, Phys. Rev. Lett. 77, 274 (1996).

[4] B. Miller, C.O. Hern, and R.P. Behringer, Phys. Rev. Lett. 77, 3110 (1996).

[5] J. Biarez and K. Wiendieck, C. R. Acad. Sci. 256, 1217 (1963).

[6] M. Oda, Soils Foundations 12, No. 2, 2 (1972).

[7] C. Thornton and D. J. Barnes, Acta Mech. 64, 45 (1986).

[8] L. Rothenburg and R. J. Bathurst, Geotechnique 39, No. 4, 601 (1989).

[9] B. Cambou, in Powders and Grains 93, edited by C. Thornton (A. A. Balkema, Rotterdam, 1993), p. 73.

[10] P.S. Cundall and O.D.L. Strack, in Mechanics of Granular Materials: New Models and Constitutive Relations, edited by J. T. Jenkins and M. Satake (Elsevier, Amsterdam, 1983).

[11] J. J. Moreau, Eur. J. Mech. A, Solids 13, No. 4-Suppl., 93-114 (1994); M. Jean, in Mechanics of Geometrical Interfaces, edited by A.P.S. Selvadurai and M. J. Boulon (Elsevier Science B.V., Amsterdam, 1995).

[12] This is in general true for simple histories of deformation. Cases where the second deviatoric component becomes important have been reported [7,9].

[13] J. Christoffersen, M. M. Mehrabadi, and S. Nemat-Nasser, J. Appl. Mech. 48, 339 (1981).

[14] F. Radjai, L. Brendel, and S. Roux, Phys. Rev. E 54, 861 (1996).

[15] F. Radjai, D. E. Wolf, and J. J. Moreau (unpublished). 